\title{
Verzeichnis der Briefe
}

1. An Charlotte von Stein, 18. September $1786 \ldots \ldots . \ldots .3$

2. An Johann Gottfried und Caroline Herder, $\langle 18$. September 1786〉.................. 3

3. An Carl August Herzog von Sachsen-Weimar und Eisenach, $\langle 18$. September 1786〉.............. 4

4. An Christian Gottlob Voigt, 〈18. September 1786〉 .... . 7

5. An Philipp Seidel, 18. September $1786 \ldots \ldots \ldots . \ldots 9$

6. An Charlotte von Stein, $\langle 14$. Oktober 1786〉 ........ 9

7. An Johann Gottfried Herder, $\langle 14$. Oktober 1786〉 ...... 11

8. An Carl August Herzog von Sachsen-Weimar und Eisenach, $\langle 14$. Oktober 1786 . . . . . . . . . . . . 12

9. An Philipp Seidel, 14. Oktober $\langle 1786\rangle \ldots \ldots \ldots \ldots$

10. An den Freundeskreis in Weimar, 1. November 1786 ... 14

11. An Carl August Herzog von Sachsen-Weimar und Eisenach, 3. November $1786 \ldots \ldots \ldots \ldots \ldots$

12. An Philipp Seidel, 4. November $\langle 1786\rangle \ldots \ldots \ldots \ldots \ldots$

13. An Catharina Elisabeth Goethe, 4. November 1786 ..... 18

14. An den Freundeskreis in Weimar, 7. November 1786 .... 19

15. An Charlotte von Stein, 7.-11. November 1786 . . . . . 21

16. An Friedrich von Stein, 〈zwischen 7. und 11.? November 1786〉 .......... 23

17. An Johann Gottfried und Caroline Herder, 10. und 11. November $\langle 1786\rangle \ldots \ldots \ldots \ldots \ldots \ldots$

18. An Christoph Martin Wieland, 17. November $\langle 1786\rangle \ldots .26$

19. An Carl Ludwig von Knebel, 17. November $\langle 1786\rangle \ldots . .28$

20. An Charlotte von Stein, 15. und 17./18. November $\langle 1786\rangle \ldots \ldots \ldots \ldots \ldots \ldots$

21. An den Freundeskreis in Weimar, 22. November 1786 ... 32

22. An Charlotte von Stein, 24. November 1786 ........ 34

23. An Philipp Christoph Kayser, 25. November 1786...... 36

24. An den Freundeskreis in Weimar, 2. Dezember 1786 . . . 37

25. An Charlotte von Stein, 2. Dezember 1786 .......... 39

26. An Johann Gottfried und Caroline Herder,

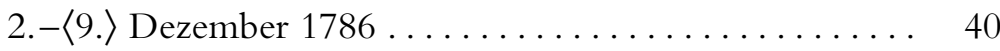


27. An den Freundeskreis in Weimar, 〈wahrscheinlich zwischen 2. und 9. Dezember 1786〉... 45

28. An Charlotte von Stein, 8. und 9. Dezember 1786 . . . . 45

29. An Philipp Seidel, 9. Dezember $\langle 1786\rangle \ldots \ldots \ldots \ldots \ldots . \ldots 4$

30. An Charlotte von Stein, 12. Dezember $1786 \ldots \ldots \ldots . .48$

31. An Philipp Seidel, 13. Dezember $1786 \ldots . \ldots \ldots \ldots . . .49$

32. An Charlotte von Stein, 〈zwischen 11. November und 16.? Dezember 1786〉.... 50

33. An Carl August Herzog von Sachsen-Weimar und Eisenach, 12.-16. Dezember 1786

34. An Johann Gottfried und Caroline Herder und deren Kinder Gottfried, August, Wilhelm, Adelbert, Luise und Emil, 13. und 16. Dezember 1786 .......... 54

35. An Charlotte von Stein, 13.-16. Dezember 1786 . . . . . 56

36. An Charlotte von Stein, $\langle$ vermutlich 16. Dezember 1786〉................. 59

37. An Louise Herzogin von Sachsen-Weimar und Eisenach, $\langle 12 . ?\rangle-23$. Dezember $1786 \ldots \ldots \ldots \ldots \ldots . . . . .59$

38. An Charlotte von Stein, 20.-23. Dezember 1786 . . . . . 61

39. An Friedrich von Stein, 29. Dezember 1786 . . . . . . . . 64

40. An Charlotte von Stein, 29. und 30. Dezember 1786 . . . 65

41. An Johann Gottfried Herder, 29. und 30. Dezember $1786 \ldots \ldots \ldots \ldots \ldots \ldots \ldots$

42. An Philipp Seidel, 30. Dezember $\langle 1786\rangle \ldots \ldots \ldots \ldots \ldots$

43. An Friedrich von Stein, 4. Januar $1787 \ldots \ldots \ldots \ldots \ldots . . . . .71$

44. An Charlotte von Stein, 6. Januar $1787 \ldots \ldots \ldots \ldots$

45. An Johann Georg Schlosser, 11. Januar $\langle 1787\rangle \ldots . . \ldots . .77$

46. An Johann Gottfried Herder, 13. Januar 1787 . . . . . . . 78

47. An Georg Joachim Göschen, 13. Januar 1787 . . . . . . . . 79

48. An Philipp Seidel, 〈13. Januar 1787〉 . . . . . . . . . . 80

49. An den Freundeskreis in Weimar, 13. Januar 1787 ... . . . 81

50. An Charlotte von Stein, 13. Januar $1787 \ldots \ldots \ldots . \ldots 8$

51. An Johann Gottfried Herder, 13. Januar 1787 . . . . . . . 83

52. An Christian Gottlob Heyne, 13. Januar 1787 . . . . . . . 85

53. An Friedrich Heinrich Jacobi, 13. Januar 1787 . . . . . . 86

54. An Philipp Christoph Kayser, 13. Januar 1787 . . . . . . . 88 
55. An Carl August Herzog von Sachsen-Weimar und Eisenach, $\langle 13\rangle-$.20 . Januar $\langle 1787\rangle \ldots \ldots \ldots \ldots \ldots \ldots$

56. An Charlotte von Stein, 17.-20. Januar 1787 . . . . . . . 91

57. An den Freundeskreis in Weimar, 25. Januar 1787 ..... 96

58. An Johann Gottfried Herder, 25. Januar 1787 . . . . . . . 97

59. An Charlotte von Stein, 25.-27. Januar $1787 \ldots \ldots \ldots . .100$

60. An Charlotte von Stein, 1.-3. Februar $1787 \ldots \ldots \ldots \ldots$

61. An Johann Gottfried und Caroline Herder, 3. Februar 1787 . . . . . . . . . . . . . . . . . . . . 107

62. An Christian Gottlob Voigt, 3. Februar 1787 . . . . . . . 109

63. An Philipp Seidel, 3. Februar 1787 . . . . . . . . . . . 110

64. An Carl August Herzog von Sachsen-Weimar und Eisenach, 3. Februar $1787 \ldots \ldots \ldots \ldots \ldots \ldots \ldots$

65. An Ernst II. Ludwig Herzog von Sachsen-Gotha und Altenburg, 6. Februar $1787 \ldots \ldots \ldots \ldots \ldots$

66. An Philipp Christoph Kayser, 6. Februar 1787 . . . . . . 115

67. An Charlotte von Stein, 〈7.-10.?〉 Februar 1787 . . . . . 116

68. An Carl August Herzog von Sachsen-Weimar und Eisenach, 10. Februar 1787 . . . . . . . . . . . . . . . 119

69. An Johann Heinrich Merck, 10. Februar 1787 . . . . . . . 121

70. An Charlotte von Stein, 13. und 17. Februar 1787 ..... 121

71. An Johann Gottfried Herder, 17. Februar $\langle 1787\rangle \ldots . . . \quad 125$

72. An Philipp Seidel, 17. Februar $\langle 1787\rangle \ldots \ldots \ldots \ldots \ldots . \ldots 127$

73. An Johann Christian Kestner, 19. Februar 1787 . . . . . . 129

74. An Carl Ludwig von Knebel, 19. Februar 1787 . . . . . . . 129

75. An Jacob Friedrich von Fritsch, 20. Februar 1787 ..... 131

76. An Georg Joachim Göschen, 20. Februar 1787 . . . . . . 133

77. An Philipp Seidel, 20. Februar $1787 \ldots \ldots \ldots \ldots \ldots$

78. An Charlotte von Stein, 19. und 21. Februar 1787 ..... 137

79. An Philipp Seidel, 〈wahrscheinlich zwischen 19. und 21. Februar 1787〉... 139

80. An Charlotte von Stein, 21. Februar $1787 \ldots \ldots \ldots \ldots$

81. An Philipp Seidel, 3. März $\langle 1787\rangle \ldots \ldots \ldots \ldots \ldots \ldots . \ldots . . \ldots 140$

82. An Friedrich von Stein, 10. März $1787 \ldots \ldots \ldots \ldots \ldots . . .141$

83. An Christian Gottlob Voigt, 23. März 1787 . . . . . . . . 142

84. An Friedrich von Stein, 〈17.〉 April $1787 \ldots \ldots \ldots \ldots . . .143$

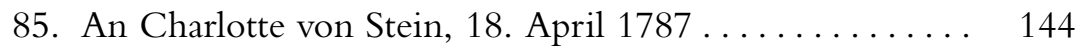


86. An Philipp Seidel, 15. Mai $1787 \ldots \ldots \ldots \ldots \ldots \ldots$

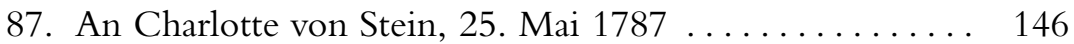

88. An Friedrich von Stein, 26. Mai $1787 \ldots \ldots \ldots \ldots \ldots$

89. An Carl August Herzog von Sachsen-Weimar und Eisenach, 27. Mai 1787 . . . . . . . . . . . . . . 150

90. An Charlotte von Stein, 1. und 8. Juni 1787 . . . . . . . . 154

91. An Gottfried, August, Wilhelm, Adelbert, Luise und Emil Herder sowie Friedrich von Stein, 30. Juni 1787 . . 158

92. An Carl August Herzog von Sachsen-Weimar und Eisenach, 6. und $\langle 7$.$\rangle Juli 1787 \ldots \ldots$. . . . . . . . . . . . 159

93. An Philipp Christoph Kayser, 14. Juli 1787 . . . . . . . . 161

94. An Charlotte von Stein, 〈28.? Juli 1787〉 . . . . . . . . . . . . 161

95. An Carl August Herzog von Sachsen-Weimar und Eisenach, 11. August $1787 \ldots \ldots \ldots \ldots$. . . . . . . . . 163

96. An Philipp Christoph Kayser, 14. August 1787 . . . . . . . 165

97. An Georg Joachim Göschen, 15. August 1787 . . . . . . . 167

98. An Charlotte von Stein, 〈wahrscheinlich zwischen 29. Juli und 18. August 1787 . . . . . . . . . . . . 169

99. An Johanne Susanne Bohl, 18. August 1787 . . . . . . . . 169

100. An Carl Ludwig von Knebel, 18. August 1787 . . . . . . . 172

101. An Philipp Seidel, 18. August $\langle 1787\rangle \ldots \ldots \ldots \ldots \ldots$

102. An Charlotte von Stein, 〈vermutlich zwischen 19. und 25. August 1787〉 ...... 175

103. An Philipp Christoph Kayser, 11. September 1787 . . . . . 176

104. An den Freundeskreis in Weimar, 17. September 1787 . . 177

105. An Carl August Herzog von Sachsen-Weimar und Eisenach, 28. September $1787 \ldots \ldots \ldots \ldots \ldots \ldots \ldots 1$

106. An Christian Friedrich Schnauß?, 1. Oktober 1787 .... 183

107. An Carl Ludwig von Knebel, 3. Oktober 1787 . . . . . . . 185

108. An Carl August Herzog von Sachsen-Weimar und Eisenach, 23. Oktober $1787 \ldots \ldots \ldots \ldots \ldots \ldots$. . . . . 187

109. An Christian Gottlob Voigt, 23. Oktober 1787 . . . . . . . 189

110. An Johann Christian Kestner, 24. Oktober 1787 . . . . . . . 191

111. An Daniel Wilhelm Brunnquell, 27. Oktober 1787 ... . 192

112. An Jacob Friedrich von Fritsch, 〈27.〉 Oktober 1787 . . . . 192

113. An Philipp Seidel, 〈27.〉 Oktober 1787 . . . . . . . . . . . 194

114. An Georg Joachim Göschen, 〈27. Oktober 1787〉 . . . . . 196 
115. An Friedrich Justin Bertuch, 〈27.〉 Oktober 1787 . . . . . 201

116. An Karl August von Hardenberg, 3. November 1787 . . . 202

117. An Philipp Seidel, $\langle$ zwischen 27. Oktober und 10. November 1787〉 ..... 203

118. An Friedrich Hildebrand von Einsiedel, 10. November $1787 \ldots \ldots \ldots \ldots$. . . . . . . . . . 205

119. An Philipp Seidel, 10. November $1787 \ldots \ldots \ldots \ldots \ldots . . \ldots 206$

120. An Philipp Seidel, 12. November 1787 . . . . . . . . . . 208

121. An Carl August Herzog von Sachsen-Weimar und Eisenach, 17. November 1787 . . . . . . . . . . . . 208

122. An Johann August Ludecus, 17. November 1787 . . . . . . 212

123. An Philipp Seidel, 17. November 1787 . . . . . . . . . 213

124. An Carl August Herzog von Sachsen-Weimar und Eisenach, 7. und 8. Dezember $1787 \ldots \ldots \ldots \ldots \ldots . \ldots 214$

125. An Philipp Seidel, 〈8.? Dezember 1787〉 . . . . . . . . 218

126. An Charlotte von Stein, 15. Dezember 1787 . . . . . . . 219

127. An Friedrich von Stein, 18. Dezember $1787 \ldots . . . \ldots .220$

128. An Carl Ludwig von Knebel, 21. Dezember 1787 . . . . . 221

129. An Philipp Seidel, 21. Dezember $1787 \ldots \ldots \ldots \ldots . \ldots 22$

130. An Christian Gottlob Voigt, $\langle$ zwischen 23. und 29. Dezember 1787〉 ......... 223

131. An Carl August Herzog von Sachsen-Weimar und Eisenach, 29. Dezember $1787 \ldots \ldots \ldots \ldots \ldots \ldots \ldots$

132. An Philipp Seidel, 〈29.? Dezember 1787〉 . . . . . . . . 227

133. An Philipp Seidel, 5. Januar 1788 . . . . . . . . . . . . . 229

134. An Charlotte von Stein, 19. Januar $\langle 1788\rangle \ldots . . . . . . . .229$

135. An Carl August Herzog von Sachsen-Weimar und Eisenach, 25. Januar $1788 \ldots \ldots \ldots \ldots \ldots \ldots 232$

136. An Charlotte von Stein, 26. Januar $\langle 1788\rangle \ldots \ldots \ldots \ldots . .240$

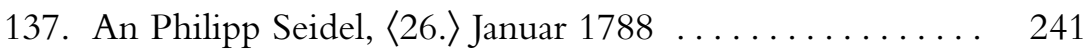

138. An Christian Gottlob Voigt,

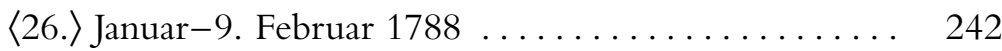

139. An Georg Joachim Göschen, 9. Februar 1788 . . . . . . . 244

140. An Philipp Seidel, 9. Februar 1788 ............. 246

141. An Carl August Herzog von Sachsen-Weimar und Eisenach, 16. Februar $\langle 1788\rangle \ldots \ldots \ldots \ldots \ldots \ldots \ldots$

142. An Friedrich von Stein, 16. Februar $1788 \ldots \ldots \ldots \ldots . .250$ 
143. An Philipp Seidel, 〈zwischen 12. und 15. März 1788〉.. 254

144. An Carl August Herzog von Sachsen-Weimar und Eisenach, 17. und 18. März $1788 \ldots \ldots \ldots \ldots \ldots \ldots$

145. An Georg Joachim Göschen, 21. März 1788 . . . . . . . 260

146. An Jacob Friedrich von Fritsch, 〈24. März 1788 . . . . . 260

147. An Christian Friedrich Schnauß, 24. März $\langle 1788\rangle$.... . 261

148. An Carl August Herzog von Sachsen-Weimar und Eisenach, 28. März-2. April 1788 . . . . . . . . . . 263

149. An Georg Joachim Göschen, 5. April 1788 . . . . . . . 265

150. An Friedrich Justin Bertuch, 5. April 1788 . . . . . . . 266

151. An Angelika Kauffmann, 〈wahrscheinlich zwischen Ende Oktober 1787 und Mitte April 1788〉......... 268

152. An Philipp Seidel, 19. April $1788 \ldots \ldots \ldots \ldots \ldots . \ldots . . \ldots 268$

153. An Carl August Herzog von Sachsen-Weimar und Eisenach, 6. Mai $1788 \ldots \ldots \ldots \ldots \ldots \ldots \ldots$

154. An Carl August Herzog von Sachsen-Weimar und

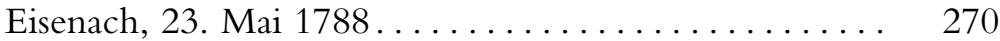

155. An Carl Ludwig von Knebel, 24. Mai $1788 \ldots . . . . . . .273$

156. An Johann Gottfried Herder,

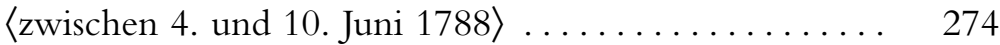

\section{Erschlossene Briefe}

EB 1. An Carl Ludwig von Knebel, 〈14.? Oktober 1786〉..

EB 2. An die Gebrüder Bethmann, 〈3. oder 4. November 1786〉.

EB 3. An Carl August Herzog von Sachsen-Weimar und Eisenach, $\langle 17$. oder 18. November 1786〉 ........ 280

EB 4. An Barbara Schultheß, 〈zwischen 22.? und 25. November 1786〉 .........

EB 5. An Anna Amalia Herzogin von Sachsen-Weimar und Eisenach, 〈zwischen 12.? und 16. Dezember 1786〉..

EB 6. An August Friedrich Standtke, $\langle$ zwischen 12.? und 16. Dezember 1786〉.........

EB 7. An Joachim Heinrich Campe, $\langle$ zwischen 12.? und 16. Dezember 1786〉. 
EB 8. An Sylvius Friedrich Ludwig von Franckenberg und Ludwigsdorf, 〈zwischen 17. und 23. Dezember 1786〉

EB 9. An August Prinz von Sachsen-Gotha und Altenburg, 〈zwischen 17. und 23. Dezember 1786〉......... 283

EB 10. An Christian Konrad Wilhelm von Dohm, $\langle$ zwischen 17. und 23. Dezember 1786〉..........

EB 11. An Sophia Amalie Erdmuthe Standtke, $\langle$ zwischen 17. und 23. Dezember 1786〉.........

EB 12. An Joachim Heinrich Campe, 〈zwischen 17. und 23. Dezember 1786〉........ 284

EB 13. An Barbara Schultheß, 〈29. oder 30. Dezember 1786〉 284

EB 14. An Catharina Elisabeth Goethe, 〈11. und/oder 12./13. Januar 1787〉 . . . . . . . . 284

EB 15. An Johann Jakob Heinrich Paulsen, 〈13.? Januar 1787〉 284

EB 16. An August Prinz von Sachsen-Gotha und Altenburg, $\langle 13$. ? Januar 1787〉 . . . . . . . . . . . . . . . . 285

EB 17. An Sophia Amalie Erdmuthe Standtke, $\langle 13$. ? Januar 1787〉 . . . . . . . . . . . . . . . . . 285

EB 18. An Wilhelm von Edelsheim, 〈20.? Januar 1787〉 . . . 285

EB 19. An Wilhelm von Edelsheim, 〈3.? Februar 1787〉 .... 286

EB 20. An Martin Ernst von Schlieffen?, $\langle$ zwischen 4. und 10. Februar 1787〉........ 286

EB 21. An Barbara SchultheB, $\langle$ zwischen 6.? und 10. Februar 1787〉 ..........

EB 22. An Christian Friedrich Schnauß, $\langle$ zwischen 7.? und 10. Februar 1787〉 . . . . . . 287

EB 23. An Louise von Göchhausen, $\langle$ zwischen 7.? und 10. Februar 1787〉 ........ 287

EB 24. An Philipp Seidel, $\langle$ zwischen 7.? und 10. Februar 1787〉 ........ 288

EB 25. An Wilhelm von Edelsheim, 〈10.? Februar 1787〉.... 288

EB 26. An Johann Christoph Schmidt, 〈20.? Februar 1787〉.. 288

EB 27. An Johann Christian Gülicke, 〈20.? Februar 1787〉 ... 289

EB 28. An Franz Ludwig Ernst Albrecht von Hendrich, $\langle 20$. ? Februar 1787 . . . . . . . . . . . . . . . 289

EB 29. An Charlotte von Stein, 〈26. oder 27. Februar 1787〉.. 289 
EB 30. An Carl Ludwig von Knebel, 〈zwischen 25. Februar und 1.? März 1787〉 . . . . . . 290

EB 31. An Carl Ludwig von Knebel, 〈zwischen 25. Februar und 1.? März 1787〉 . . . . . . 290

EB 32. An Johann Gottfried Herder, 〈3.? März 1787〉 . . . . . 290

EB 33. An Charlotte von Stein, 〈zwischen 4. und 10. März 1787〉. . . . . . . . . . 291

EB 34. An Charlotte von Stein, 〈zwischen 14. und 16. März 1787〉............ 291

EB 35. An Carl August Herzog von Sachsen-Weimar und Eisenach, 〈zwischen 14. und 16. März 1787〉 . . . . . 291

EB 36. An Caroline Herder,

〈zwischen 14. und 16. März 1787〉...........

EB 37. An Philipp Seidel,

〈zwischen 14. und 16. oder am 17. März 1787〉 . . . 292

EB 38. An Charlotte von Stein, 〈19. und/oder 20. März 1787〉 292

EB 39. An Friedrich von Stein, 〈19. und/oder 20. März 1787〉 292

EB 40. An Angelika Kauffmann, 〈20. März 1787〉 . . . . . . 292

EB 41. An Johann Friedrich Reiffenstein, 〈20. März 1787〉. . 292

EB 42. An Charlotte von Stein, 〈zwischen 21. und 27. März 1787〉 . . . . . . . . 293

EB 43. An Carl August Herzog von Sachsen-Weimar und Eisenach, 〈zwischen 21. und 27. März 1787〉 . . . . 293

EB 44. An Barbara Schultheß, 〈zwischen 21. und 27. März 1787〉............

EB 45. An Christian Gottlob Heyne, 〈zwischen 21. und 27. März 1787〉............

EB 46. An Friedrich Wilhelm Heinrich von Trebra, 〈zwischen 21. und 27. März 1787〉............

EB 47. An Joseph Friedrich von Racknitz, 〈zwischen 21. und 27. März 1787〉 . . . . . . . . . .

EB 48. An Carl Ludwig von Knebel, 〈zwischen 21.? und 27.? März 1787〉 . . . . . . . . . 294

EB 49. An Carl Ludwig von Knebel, 〈zwischen 22.? und 29.? März 1787〉 . . . . . . . . . 295

EB 50. An Charlotte von Stein, 〈28. oder 29. März 1787〉... 295 
EB 51. An Charlotte von Stein, $\langle$ zwischen 1. und 5. April 1787〉 ............ 295

EB 52. An Charlotte von Stein, 〈zwischen 6. und 12. April 1787〉 ........... 296

EB 53. An Jakob Philipp Hackert, 〈zwischen 6. und 12. April 1787〉 ........... 296

EB 54. An Johann Heinrich Wilhelm Tischbein, 〈17. oder 18. April 1787〉 . . . . . . . . . . . . 296

EB 55. An Jakob Philipp Hackert, 〈17. oder 18. April 1787〉 . . 297

EB 56. An August Prinz von Sachsen-Gotha und Altenburg, 〈zwischen 9. und 11. Mai 1787〉 .............. 297

EB 57. An Sophie von Lichtenberg?,〈14. oder 15. Mai 1787〉 297

EB 58. An Charlotte von Stein, 〈14. oder 15. Mai 1787〉.... 298

EB 59. An den Freundeskreis in Weimar, $\langle 14$. oder 15. Mai 1787〉 . . . . . . . . . . . . . . . . . . 298

EB 60. An Angelika Kauffmann, 〈14. oder 15. Mai 1787〉... 298

EB 61. An Johann Heinrich Wilhelm Tischbein, $\langle 14$. oder 15. Mai 1787〉 . . . . . . . . . . . . .

EB 62. An Anna Amalia Herzogin von Sachsen-Weimar und Eisenach, 〈zwischen 25.? und 29. Mai 1787〉 . . . . . . .

EB 63. An Johann Gottfried Herder, 〈zwischen 25.? und 29. Mai 1787〉 ..............

EB 64. An Barbara Schultheß, $\langle$ zwischen 25.? und 29. Mai 1787〉 ............

EB 65. An Charlotte von Stein, $\langle$ zwischen 30. Mai und 2. Juni 1787〉 ...........

EB 66. An Charlotte von Stein, $\langle$ zwischen 17.? und 23. Juni 1787〉 . . . . . . . . . 300

EB 67. An Johann Gottfried Herder, 〈zwischen 17.? und 23. Juni 1787〉 . . . . . . . . . 300

EB 68. An Carl Theodor von Dalberg?, 〈zwischen 17.? und 23. Juni 1787〉 . . . . . . . . 300

EB 69. An Catharina Elisabeth Goethe, $\langle$ zwischen 17.? und 23. Juni 1787〉 . . . . . . . . 301

EB 70. An Charlotte von Stein, 〈30.? Juni 1787〉 . . . . . . . 301

EB 71. An Johann Gottfried Herder, 〈30.? Juni 1787〉 . . . . . 301

EB 72. An Charlotte von Stein, 〈zwischen 1. und 7. Juli 1787〉 301 
EB 73. An Johann Heinrich Wilhelm Tischbein, 〈zwischen 8.? und 13. Juli 1787〉 .......... 302

EB 74. An Barbara Schultheß, 〈14.? Juli 1787〉 . . . . . . . . 302

EB 75. An Charlotte von Stein, 〈14.? Juli 1787〉 . . . . . . . 302

EB 76. An Anna Amalia Herzogin von Sachsen-Weimar und Eisenach, 〈zwischen 15. und 21. Juli 1787〉 . . . . . 302

EB 77. An Charlotte von Stein, $\langle$ zwischen 15. und 21. Juli 1787〉 ........... 303

EB 78. An Johann Gottfried Herder, $\langle$ zwischen 15. und 21. Juli 1787〉 ........... 303

EB 79. An Johann Heinrich Wilhelm Tischbein, $\langle$ zwischen 15. und 21. Juli 1787〉 ........... 303

EB 80. An Charlotte von Stein, $\langle$ zwischen 29. Juli und 4. August 1787〉........ 303

EB 81. An Charlotte von Stein, $\langle$ zwischen 5. und 11. August 1787〉 ......... 303

EB 82. An Johann Gottfried Herder, 〈zwischen 5. und 11. August 1787〉 . . . . . . . . 304

EB 83. An Anna Amalia Herzogin von Sachsen-Weimar und Eisenach, 〈zwischen 5. und 11. August 1787〉..... 304

EB 84. An Johann Heinrich Wilhelm Tischbein, $\langle$ zwischen 12.? und 14. August 1787〉 . . . . . . . . 304

EB 85. An Joseph Johann Reichsgraf von Fries, $\langle$ zwischen 12.? und 14. August 1787〉 .......... 304

EB 86. An Johann Jakob Heinrich Paulsen, 〈zwischen 6.? Juni und 18. August 1787〉 . . . . . . 304

EB 87. An August Prinz von Sachsen-Gotha und Altenburg, 〈zwischen 12. und 18. August 1787〉 ........... 305

EB 88. An Friedrich von Stein, 〈zwischen 12. und 18. August 1787〉......... 305

EB 89. An Charlotte von Stein, 〈zwischen 12. und 18. August 1787〉........ 305

EB 90. An Barbara Schultheß, 〈zwischen 14.? und 18. August 1787〉 ......... 305

EB 91. An Anna Amalia Herzogin von Sachsen-Weimar und Eisenach, 〈18.? August 1787〉 . . . . . . . . . . . . . . 306 
EB 92. An Barbara Schultheß, $\langle$ zwischen 19. und 25.? August 1787〉 . . . . . . 306

EB 93. An Charlotte von Stein, 〈zwischen 2.? und 8. September 1787〉 . . . . . . . 306

EB 94. An Barbara Schultheß, $\langle$ zwischen 2.? und 8. September 1787〉 . . . . . . 306

EB 95. An Charlotte von Stein, $\langle$ zwischen 9. und 15. September 1787〉........ 307

EB 96. An Johann Gottfried und Caroline Herder, $\langle$ zwischen 9. und 15. September 1787〉........ 307

EB 97. An Johann Gottfried Herder, 〈15.? September 1787〉 . . 307

EB 98. An Charlotte von Stein, $\langle$ zwischen 16. und 22. September 1787〉....... 307

EB 99. An Johann Gottfried Herder, $\langle$ zwischen 16. und 22. September 1787〉....... 308

EB 100. An Anna Amalia Herzogin von Sachsen-Weimar und Eisenach, 〈zwischen 16. und 22. September 1787〉 . . 308

EB 101. An Catharina Elisabeth Goethe, $\langle$ zwischen 16. und 22. September 1787〉....... 308

EB 102. An Georg Melchior Kraus, 〈zwischen 6?. Juni und 25.? September 1787〉 . . . . 308

EB 103. An Johann Gottfried Herder, 〈6. Oktober 1787〉 . . . . 309

EB 104. An Johann Gottfried Herder, $\langle$ zwischen 7. und 12. Oktober 1787〉 .......... 309

EB 105. An Charlotte von Stein, $\langle$ zwischen 7. und 12. Oktober 1787〉 .......... 310

EB 106. An Johann Gottfried Herder, 〈23.? Oktober 1787〉 . . 310

EB 107. An Anna Amalia Herzogin von Sachsen-Weimar und Eisenach, $\langle 23$. ? Oktober 1787〉 . . . . . . . . . . . 310

EB 108. An Johann Christoph Schmidt, $\langle$ zwischen 23. und 27. Oktober 1787〉 ........ 311

EB 109. An Barbara Schultheß, $\langle$ zwischen 23. und 27. Oktober 1787〉 ..........

EB 110. An Catharina Elisabeth Goethe, 〈zwischen 23. und 27. Oktober 1787〉 ......... 311

EB 111. An Christian Gottfried Schütz?, $\langle$ zwischen 23. und 27. Oktober 1787〉 .......... 
EB 112. An Ernst II. Ludwig Herzog von Sachsen-Gotha und Altenburg, 〈zwischen 23. und 27. Oktober 1787〉 ...

EB 113. An Charlotte von Stein, $\langle$ zwischen 4. und 10. November 1787〉 . . . . . . . .

EB 114. An Johann Gottfried Herder, $\langle$ zwischen 4. und 10. November 1787〉 . . . . . . . . .

EB 115. An Louise von Göchhausen, $\langle$ zwischen 4. und 10. November 1787〉 . . . . . . . . .

EB 116. An Anna Amalia Herzogin von Sachsen-Weimar und Eisenach, 〈zwischen 4. und 10. November 1787〉....

EB 117. An Sylvius Friedrich Ludwig von Franckenberg und Ludwigsdorf, 〈zwischen 4. und 10. November 1787〉. .

EB 118. An Louise Herzogin von Sachsen-Weimar und Eisenach, $\langle 17$. ? November 1787〉 . . . . . . . . . . . . . .

EB 119. An Charlotte von Stein, 〈17.? November 1787〉 . . . .

EB 120. An Ludwig Ernst Herzog von Braunschweig und Lüneburg-Wolfenbüttel?, 〈17.? November 1787〉 . . . .

EB 121. An Catharina Elisabeth Goethe, $\langle 17$. ? November 1787 . . . . . . . . . . . . . . . . . . .

EB 122. An Charlotte von Stein, 〈zwischen 18. und 24. November 1787〉.........

EB 123. An Charlotte von Stein, $\langle$ zwischen 25. November und 1. Dezember 1787〉...

EB 124. An Johann Gottfried Herder, $\langle$ zwischen 25. November und 1. Dezember 1787〉...

EB 125. An Charlotte von Stein,

$\langle$ zwischen 2. und 8. Dezember 1787〉 ..........

EB 126. An Friedrich von Stein,

$\langle$ zwischen 2. und 8. Dezember 1787〉 ...........

EB 127. An Johann Christian Stark,

$\langle$ zwischen 2. und 8. Dezember 1787〉 ..........

EB 128. An Charlotte von Stein,

$\langle$ zwischen 9. und 15. Dezember 1787〉 ........ 316

EB 129. An Charlotte von Stein, 〈21.? Dezember 1787〉 . . . .

EB 130. An Anna Amalia Herzogin von Sachsen-Weimar und Eisenach, $\langle 21$. ? Dezember 1787〉 . . . . . . . . . . . . 
EB 131. An Carl Wilhelm Thurneysen, $\langle$ zwischen 16. und 22. Dezember 1787〉..........

EB 132. An Charlotte von Stein, $\langle$ zwischen 23. und 29. Dezember 1787〉.........

EB 133. An Johann Gottfried Herder, $\langle$ zwischen 23. und 29. Dezember 1787〉 . . . . . . .

EB 134. An Catharina Elisabeth Goethe, $\langle$ zwischen 23. und 29. Dezember 1787〉 . . . . . . . .

EB 135. An Charlotte von Stein, $\langle$ zwischen 6.? und 12. Januar 1788〉

EB 136. An Johann Gottfried Herder, 〈zwischen 6.? und 12. Januar 1788〉 . . . . . . . . 318

EB 137. An Johann Gottfried Herder, 〈26.? Januar 1788〉 . . . . . 318

EB 138. An Anna Amalia Herzogin von Sachsen-Weimar und Eisenach, 〈zwischen 27. Januar und 2. Februar 1788〉 . .

EB 139. An Charlotte von Stein,

$\langle$ zwischen 27. Januar und 2. Februar 1788〉 ........

EB 140. An Catharina Elisabeth Goethe, 〈zwischen 27. Januar und 2. Februar 1788〉 . . . . . .

EB 141. An Johann Gottfried Herder,

$\langle$ zwischen 3. und 9. Februar 1788〉 . . . . . . . .

EB 142. An Charlotte von Stein?,

$\langle$ zwischen 3. und 9. Februar 1788〉 . . . . . . . . .

EB 143. An Filippo Collina,

$\langle$ zwischen 3. und 9. Februar 1788〉 ............

EB 144. An Friedrich Carl Kayser,

〈zwischen 3. und 9. Februar 1788〉

EB 145. An Georg Philipp Lehr?,

$\langle$ zwischen 3. und 9. Februar 1788〉 ...........

EB 146. An Friedrich Justin Bertuch,

$\langle z w i s c h e n$ 3. und 9. Februar 1788〉 ............

EB 147. An Georg Melchior Kraus,

〈zwischen 3. und 9. Februar 1788〉

EB 148. An Charlotte von Stein, 〈16.? Februar 1788〉 . . . . . . .

EB 149. An August Prinz von Sachsen-Gotha und Altenburg, 
EB 150. An Charlotte von Stein, 〈zwischen 24.? Februar und 1. März 1788〉.......

EB 151. An Johann Gottfried Herder, 〈zwischen 24.? Februar und 1. März 1788〉 . . . . . . .

EB 152. An Christoph Heinrich Kniep, 〈vermutlich zwischen Januar und Anfang März 1788〉

EB 153. An Charlotte von Stein, 〈zwischen 2. und 8. März 1788〉 . . . . . . . . . . . . .

EB 154. An Barbara Schultheß, $\langle z w i s c h e n$ 2. und 8. März 1788〉...............

EB 155. An Charlotte von Stein, $\langle$ zwischen 9. und 15. März 1788〉..............

EB 156. An Johann Gottfried Herder, 〈zwischen 9. und 15. März 1788〉............. 324

EB 157. An Catharina Elisabeth Goethe, 〈zwischen 9. und 15. März 1788〉. . . . . . . . . . 324

EB 158. An Schramm \& Kerstens, $\langle$ zwischen 9. und 15. März 1788〉.............

EB 159. An Charlotte von Stein, $\langle$ zwischen 16. und 22. März 1788〉.......... 324

EB 160. An Christoph Heinrich Kniep, 〈23. März 1788〉 . . . 325

EB 161. An Johann Christoph Schmidt, 〈24.? März 1788〉 . . 325

EB 162. An Anna Amalia Herzogin von Sachsen-Weimar und Eisenach, 〈zwischen 23. und 29. März 1788〉...

EB 163. An Ernst II. Ludwig Herzog und Charlotte Herzogin von Sachsen-Gotha und Altenburg, 〈zwischen 30. März und 5. April 1788〉.........

EB 164. An Charlotte von Stein, 〈zwischen 6. und 12. April 1788〉.............

EB 165. An August Friedrich Standtke, 〈zwischen 6. und 12. April 1788〉........... 326

EB 166. An Johann Gottfried Herder, 〈19.? April 1788〉 . . . . 326

EB 167. An Louise von Göchhausen, 〈19.? April 1788〉 . . . . . 327

EB 168. An Carl Wilhelm Thurneysen, 〈20.? April 1788〉... 327

EB 169. An Catharina Elisabeth Goethe, 〈20.? April 1788〉 . . 327

EB 170. An Johann Heinrich Wilhelm Tischbein, $\langle 20 . ?$ April 1788〉 
EB 171. An Christoph Heinrich Kniep, 〈20.? April 1788〉 . . . 328

EB 172. An Jakob Philipp Hackert, 〈20.? April 1788〉 . . . . . . 328

EB 173. An Angelika Kauffmann, 〈23.? oder 24. April 1788〉.. 328

EB 174. An Angelika Kauffmann,

〈zwischen 30. April und 7. Mai 1788〉........ 329

EB 175. An Friedrich Bury,

〈zwischen 30. April und 7. Mai 1788〉........ 329

EB 176. An Angelika Kauffmann,

〈zwischen 6.? und 11. Mai 1788〉 ........... 330

EB 177. An Friedrich Bury, 〈zwischen 12. und 17. Mai 1788〉 330

EB 178. An Angelika Kauffmann,

〈zwischen 15.? und 17. Mai 1788〉 .......... 330

EB 179. An Barbara Schultheß, 〈23. oder 24. Mai 1788〉 .... 331

EB 180. An Johann Gottfried Herder, 〈23. oder 24. Mai 1788〉 331

EB 181. An Charlotte von Stein, 〈23. oder 24. Mai 1788〉 ... 331

EB 182. An Friedrich Bury, 〈23. oder 24. Mai 1788〉 ....... 332

EB 183. An Johann Heinrich Meyer, 〈23. oder 24. Mai 1788〉 . 332

EB 184. An Karl Philipp Moritz, 〈23. oder 24. Mai 1788〉... . 332

EB 185. An Friedrich Rehberg, 〈23. oder 24. Mai 1788〉.... 333

EB 186. An Angelika Kauffmann, 〈24.? Mai 1788〉 ........ 333

EB 187. An Johann Friedrich Reiffenstein, 〈24. Mai 1788〉 ... 333

EB 188. An Albert-Guillaume Berczy, 〈24. Mai 1788〉 ...... 334

EB 189. An Anna Amalia Herzogin von Sachsen-Weimar und Eisenach, 〈zwischen 23. und 27. Mai 1788〉... 334

EB 190. An Aloys Hirt, 〈zwischen 23. und 27. Mai 1788〉.... 335

EB 191. An Johann Heinrich Wilhelm Tischbein, 〈zwischen 23. und 27. Mai 1788〉......... 335

EB 192. An Johann Georg Schütz, 〈zwischen 23. und 27. Mai 1788〉........... 335

EB 193. An Johann Martin Gruber, 〈9.? Juni 1788〉. . . . . . . 335 
